\title{
Parallelization of Pricing Path-Dependent Financial Instruments on Bounded Trinomial Lattices
}

\author{
Hannes Schabauer ${ }^{1}$, Ronald Hochreiter ${ }^{2}$, and Georg Ch. Pflug ${ }^{2}$ \\ ${ }^{1}$ Research Lab Computational Technologies and Applications, University of Vienna \\ hannes.schabauer@univie.ac .at \\ ${ }^{2}$ Department of Statistics and Decision Support Systems, University of Vienna \\ \{ronald.hochreiter, georg.pflug\}@univie.ac.at
}

\begin{abstract}
Complex financial instruments are a central concept for the survival of financial enterprises in liberalized markets. The need for fast pricing of more complex and exotic financial products led to the development of new algorithms, and to the parallelization of existing algorithms. In this paper, we present a parallelization scheme for pricing path-dependent interest rate products on bounded trinomial lattices. The basic building block presented in this paper can be used to build more complex pricing schemes. The paper is concluded by a set of numerical results concerning the speedup of the proposed parallelization scheme.
\end{abstract}

Keywords: computational finance, pricing, bounded lattice, cluster computing, parallelization.

\section{Introduction}

Pricing a financial contract is based on the calculation of its respective present value, which is done by computing the expected, discounted cash-flow given a cash-flow function. The underlying interest rate process, which is the basis of the calculations is most commonly given as continuous-time stochastic process. In order to numerically cope with such processes, one can either apply MonteCarlo methods or use some sort of time and state discretization, see e.g. [1. If a stochastic process is simulated and discretized, then this discretization process leads to (non-recombining) trees or recombining trees (lattices) - see Figure 1 for two-stage, binomial examples of these structures.

Using a lattice model results in problems of polynomial order if the lattice has polynomial growth and cash-flows may be represented on the lattice. However, exotic contracts, which are of growing importance for practical applications, define the payable cash-flows as a function of the whole history of the underlying interest rate process. The tree covering the history grows exponentially and therefore the complexity of pricing such contracts is non-polynomial. For certain subclasses of cash-flow functions however, a polynomial pricing algorithm exists, see 2. Algorithms of this subclass are an essential component of the AURORA 


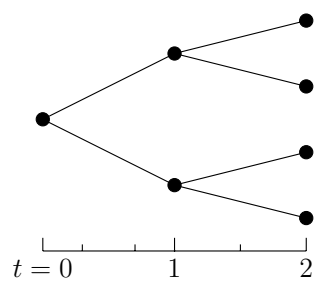

Non-recombining tree

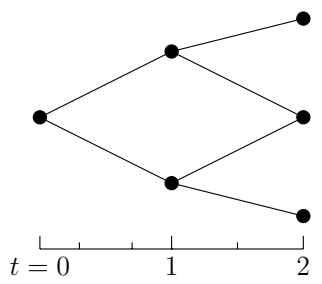

Recombining tree (lattice)

Fig. 1. Tree-type structures

Financial Management System, a modular decision support tool for asset and liability management under development at the University of Vienna, see [3]. The main aim of this paper is to present a parallelization of the algorithms presented there for the special structure of bounded trinomial lattices. For an algorithmic and implementational overview of the general pricing methodology on trees, see 4] and 11. Some foundations of the work presented in this paper have also been shown by [5]. Parallelization approaches for binomial trees (BT) have been proposed e.g. in [6] and [7]. Parallelizations of trinomial trees (TT) in [8]. In [9] two parallel - shared memory and distributed memory - algorithms for implementing the lattice methods for pricing European and American style equity options are discussed. Special parallelization schemes for Asian option structures are presented in [10].

A summary of the topic of financial pricing related to high performance computing is presented by [11. The idea is to modify pricing algorithms to optimally run on clusters of workstations and some Grid middleware (see [12] for a summary of the importance for the whole financial industry). Both extensions provide an interesting infrastructure for pricing complex financial instruments, especially with a proper portage of the underlying data format of the pricing process (trees) to specialized hardware structure.

This paper is organized as follows. Section 2 describes the lattice approach to pricing path-dependent interest rate instruments. Section 3 summarizes parallelization issues, and the chosen parallelization approach and Section 4 contains numerical results, while Section 5 concludes the paper.

\section{Path-Dependent Financial Instruments}

\subsection{Interest Rate Path Dependency}

Financial instruments tend to become increasingly complex, due to ever changing regulatory situations in liberalized markets. Many interest rate products do not only depend on the interest rate at the time of exercise of the instrument, but on an interest rate, that depends on the path of the underlying interest rate. The calculation of the effective interest rate may be based on the following modifiers. 
- Mean-type. The effective interest rate is the arithmetic mean or geometric mean of the interest rate path.

- Barrier-type. The effective interest rate is modified if the underlying interest rate reaches certain lower or upper barriers.

- Extremum-type. The effective interest rate is bound to some upper and/or lower level.

Furthermore the effective interest rate may depend on the whole path of the interest rate process (full path dependency) or only on the last $t_{l}$ stages (limited path dependency). While the effective interest rate of path-independent interest instruments depends on the current market interest rate, the contracted interest rates of path-dependent interest rate instruments are contingent to the historical path of the underlying interest rate. We assume, that a model for discretizing the process is available. Many other instruments are available respectively can be generated out of some basic building blocks. The strategy for creating complex products out of simpler building blocks is shown by [2]

The underlying time-discretized stochastic process has a finite set of $T$ stages (points in time), which constitutes the complexity parameter for the calculations. It might be useful to use a fine-grained time discretization, i.e. hourly steps. Such a fine time-discretization results in up to tens of thousands of stages for long-term financial products.

For most algorithms on lattices, a backward and a forward recursive algorithm can be defined. Introduce the conditional present values $P V_{t, j}(c)$ for a future state $(t, j)$ as the conditional expectation shown in Eq. 1 .

$$
\begin{aligned}
& \mathbb{E}\left[\sum_{s=t}^{T} c_{s}\left(\xi_{s}\right) f_{s-1}\left(\xi_{s-1}\right) \cdots f_{t}\left(\xi_{t}\right) \mid \xi_{t}=j\right]= \\
& c_{t}(j)+\mathbb{E}\left[\sum_{s=t+1}^{T} c_{s}\left(\xi_{s}\right) f_{s-1}\left(\xi_{s-1}\right) \cdots f_{t}\left(\xi_{t}\right) \mid \xi_{t}=j\right]
\end{aligned}
$$

The conditional present values $P V_{t, j}$ satisfy a recursion. The basic backward recursion to calculate the present value of a path-independent product is shown in Table 2.1. The vectors $C$ and $F$ contain the cash-flow and the discount factors, which are defined for all nodes in the lattice respectively. $S_{t}$ denotes the set of possible states at time $t$.

Table 1. Basic backward recursion algorithm

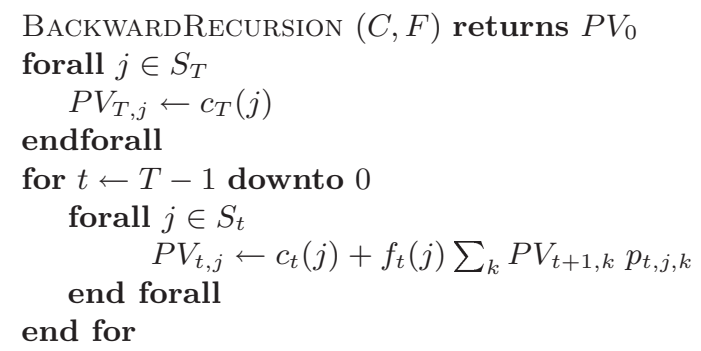


This algorithm is the basic block for pricing path-dependent present values, such as both mean-type cash flow structures, see [2].

\subsection{Bounded Trinomial Lattices}

Table 2 compares binary and ternary lattices and trees of height $T$. However, instead of working plain trinomial lattices (TL), whose number of nodes is $(T+$ $1)^{2}$, it is more common to use bounded trinomial lattices (BTL). A bounded trinomial lattice is bounded in a stage $t_{b}<T$. In all succeeding stages, starting in $t_{b}$, the highest-value as well as the lowest-value node do not branch into new additional lower and upper value nodes, i.e. the number of nodes per stage is constant $\left(\left(2 \cdot t_{b}\right)-1\right)$. A methodology to calibrate such lattices has been proposed by 13 . A degenerated bounded trinomial lattice (DBTL) has the additional feature that it is only binomial at the highest-value and lowest-value. The choice of the bound structure (degenerated or not) is determined by the underlying calibration methodology.

Table 2. Complexity of lattices and trees

\begin{tabular}{lcc}
\hline Type of lattice & Binary & Ternary \\
\hline Nodes (Lattice) & $\frac{1}{2}(T+2)(T+1)$ & $(T+1)^{2}$ \\
Arcs (Lattice) & $T(T+1)$ & $3 T^{2}$ \\
Nodes (History Tree) & $2^{T+1}-1$ & $\frac{1}{2}\left(3^{T+1}-1\right)$ \\
Arcs (History Tree) & $2^{T+1}-2$ & $\frac{1}{2}\left(3^{T+1}-3\right)$ \\
\hline
\end{tabular}

Bounded lattices consist of a triangular part and a rectangular part as shown in Figure 2. The triangular part has an increasing number of nodes and edges at each stage. The present values of both parts can be calculated in parallel. As expected, parallelization of the triangular part has turned out to be slower than parallelization of the bounded, rectangular part. The triangular part was parallelized by dynamic arrays for its nodes and edges. However, parallelization of this part is not that important, as the rectangular part is considerably larger than the triangular one in practice.

\section{Parallelization}

Our parallel approach introduces pricing of path-dependent contracts on distributed memory machines (clusters of PCs or workstations) [14.

\subsection{Iterative and Recursive Problem Pattern}

A very general algorithm for path-dependent pricing can be described as follows. The algorithm includes both bounded and non-bounded parts of a lattice. Our approach of parallelizing is a combination of a divide-and-conquer algorithm combined with data decomposition. You can imagine the structure of a path 


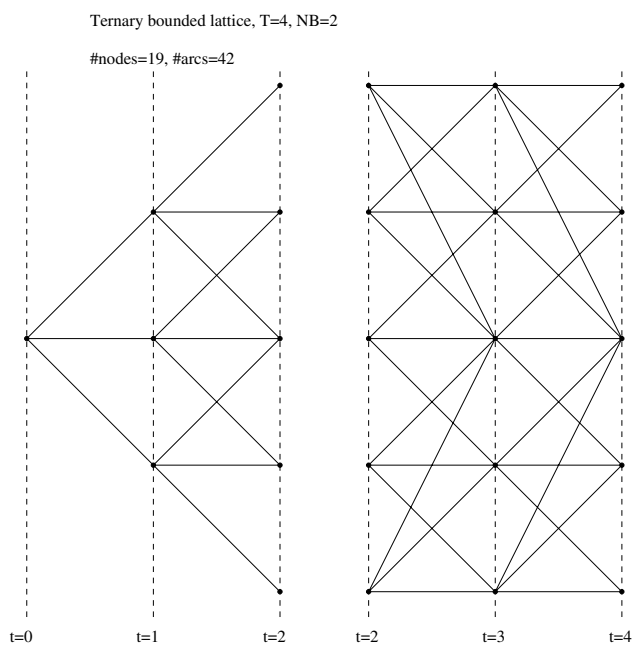

Fig. 2. Triangle and rectangle part

dependent lattice as a bundle of independent parts of the whole lattice. Therefore it is possible to calculate the parts in parallel, without communication during the computation. Each part is called here a sweep. For example, if a lattice extends over 5 periods $(T=5)$, you result in sweeps $1,2,3,4$ and 5 . Communication between the nodes is only needed before and after performing the respective sweeps. Though, one should note that each sweep has a different runtime. Our parallel algorithm divides the subproblems into parts, which can be calculated in the same amount of time together. The way of dividing the lattice into parts is as follows. Consider the example of three nested loops. The interior loop is calculating all nodes in same state, in same time period, in the same sweep. One node needs the values of all adjacent edges and vertices. These have already been calculated in the last step. Thus, in the inner loop all vertices in same period, same sweep are calculated. A covering loop iterates over all time periods start at the period of the sweep, ending at period 0 . The outer loop is iterating over all sweeps.

\subsection{Parallel Code and Data Structure}

In principle, all three loops have to be observed in order to find the best one for finding an efficient way of parallelization. Parallelization of the interior loop has been tried earlier. It turned out not to work well, as the ratio of computation to communication is not high enough. The covering loop can not be parallelized by any means, because of its recursive calculation. As described in [2, a path dependent lattice carries two different types of data: Path dependent and path independent data. Path independence occurs on discount factors and probabilities, whereas path dependency occurs on cash flows and calculated present values. From the algorithmic point of view, this means that discount factors 
and probabilities are the same for all respective sweeps. We just have to copy them before calculating. Cash flows and obviously also calculated present values are different for each iteration at each state and in each sweep. Calculating a net present value of $T$ periods yields to $T$ different subproblems (sweeps) from $1 \ldots T$. Their respective runtime complexity equals to the number of the sweep. E.g. Sweep 100 takes 50 times of sweep 2. Notice that after distributing all jobs to the nodes, we want to have our results at the end on the same time. Therefore we combine sweeps, which pairwise sum up to the same value.

\subsection{Simplified Numerical Example}

Let us consider an example of a ternary bounded lattice of height 99, lasting over 20 periods, running on a cluster with 4 nodes. Such a lattice consists of 20 distinct sweeps, starting at $1,2,3, \ldots, 19,20$. In each sweep, we need sweep $\times$ height cash flows. In order to gain the same amount of data at each sweep, we combine two of them. Here, we combine sweep 20 with sweep 1, 19 with 2 and so on. Thus we can result in 10(20/2) columns of $21 \cdot 99$ cash flows. Now we distribute these cash flows in equal sizes to our worker nodes. 10 columns for 3 worker nodes results in 3 columns per node. The remaining column is performed by the master node.

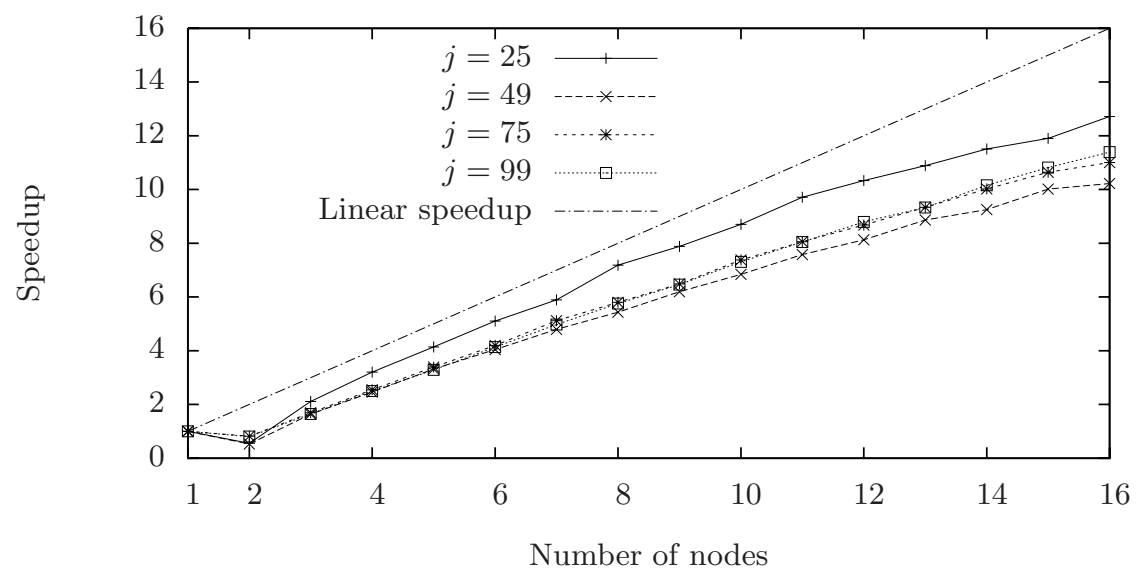

Fig. 3. Speedups for different bounded heights $j$

\section{Numerical Results}

Parallel codes have been tested on the Schroedinger III cluster 1 at the Vienna University Computer Center at the University of Vienna. Calculations were done on single-core processors, therefore all communication utilizes network communication, which takes significantly more time than locally on SMP or multi-core

\footnotetext{
1 http://www . univie.ac.at/ZID/schroedinger/
} 
Table 3. Runtime for $T=3000$ (left) and $T=2000$ (right) for $n=1$ to 16 parallel computing nodes with a bounded height $j$ of $25,49,75,99$

\begin{tabular}{rcccc} 
& 25 & 49 & 75 & 99 \\
\hline 1 & 9,487 & 15,693 & 26,778 & 35,203 \\
2 & 16,980 & 29,457 & 32,722 & 43,640 \\
3 & 4,484 & 9,566 & 15,828 & 21,339 \\
4 & 2,960 & 6,371 & 10,511 & 14,082 \\
5 & 2,289 & 4,726 & 7,890 & 10,707 \\
6 & 1,859 & 3,878 & 6,378 & 8,511 \\
7 & 1,609 & 3,273 & 5,230 & 7,070 \\
8 & 1,320 & 2,886 & 4,613 & 6,113 \\
9 & 1,203 & 2,531 & 4,128 & 5,449 \\
10 & 1,089 & 2,292 & 3,628 & 4,816 \\
11 & 0,976 & 2,070 & 3,324 & 4,375 \\
12 & 0,917 & 1,929 & 3,085 & 4,003 \\
13 & 0,871 & 1,769 & 2,867 & 3,773 \\
14 & 0,824 & 1,695 & 2,671 & 3,464 \\
15 & 0,796 & 1,566 & 2,515 & 3,253 \\
16 & 0,746 & 1,535 & 2,433 & 3,089 \\
\hline
\end{tabular}

\begin{tabular}{rcccc}
\multicolumn{1}{c}{25} & 49 & 75 & 99 \\
\hline 1 & 3,389 & 6,781 & 10,627 & 13,997 \\
2 & 5,792 & 14,789 & 24,421 & 33,812 \\
3 & 3,542 & 10,476 & 15,917 & 9,050 \\
4 & 1,316 & 2,902 & 4,539 & 5,980 \\
5 & 1,003 & 2,175 & 3,417 & 4,535 \\
6 & 0,761 & 1,722 & 2,683 & 3,574 \\
7 & 0,640 & 1,398 & 2,234 & 2,910 \\
8 & 0,535 & 1,226 & 1,914 & 2,488 \\
9 & 0,476 & 0,121 & 1,718 & 2,230 \\
10 & 0,437 & 0,988 & 1,558 & 2,003 \\
11 & 0,402 & 0,922 & 1,433 & 1,832 \\
12 & 0,421 & 0,882 & 1,386 & 1,738 \\
13 & 0,390 & 0,832 & 1,273 & 1,601 \\
14 & 0,390 & 0,785 & 1,214 & 1,519 \\
15 & 0,378 & 0,742 & 1,312 & 1,593 \\
16 & 0,363 & 0,726 & 1,558 & 1,824 \\
\hline
\end{tabular}

processors. The structure of the cluster at the time of the calculation of the results below consisted of 240 nodes. Each node has a Pentium 4 Prescott CPU, operating at $3.2 \mathrm{GHz}$, and $2 \mathrm{~GB}$ RAM. The runtime environment is operating on a Gigabit network, interconnected by a Cisco 6500 switch. The total performance is approximately 1.14 Teraflops per second (linpack).

All program codes were written in Fortran 90, using mpich 1.27. The used compiler was Portland Group pgf90 6.1-1 (x86-64 Linux).

The resulting runtime for $T=3000$ and $T=2000$ is summarized in Tab. 3 . The speedup is shown graphically for $T=3000$ for all four heights in Fig. 3 . The speedup results indicate, that the algorithm scales well.

\section{Conclusion}

In this paper, we presented a parallelization scheme for pricing path-dependent interest rate instruments defined on discrete-time bounded trinomial lattices. Generally, tree and lattice-based data structures are not perfectly suited on cluster architectures. However, the results look promising to continue the work and extend the underlying financial problems from pricing path-dependent interest rate products to option pricing or large-scale multi-stage stochastic tree-based portfolio optimization.

\section{References}

1. Jäckel, P.: Monte Carlo Methods in Finance. John Wiley \& Sons, Chichester (2002)

2. Hochreiter, R., Pflug, G.C.: Polynomial algorithms for pricing path-dependent interest rate instruments. Computational Economics 28(3), 291-309 (2006) 
3. Pflug, G.C., Świętanowski, A., Dockner, E., Moritsch, H.: The Aurora financial management system: model and parallel implementation design. Annals of Operations Research 99, 189-206 (2000)

4. Clewlow, L., Strickland, C.: Implementing Derivative Models. John Wiley \& Sons, Chichester (1998)

5. Pflug, G.C., Świętanowski, A.: Selected parallel optimization methods for financial management under uncertainty. Parallel Computing 26(1), 3-25 (2000)

6. Thulasiram, R., Bondarenko, D.: Performance evaluation of parallel algorithms for pricing multidimensional financial derivatives. In: Fourth International Workshop on High Performance Scientific and Engineering Computing with Applications (HPSECA 2002), pp. 306-313. IEEE Computer Society, Los Alamitos (2002)

7. Gerbessiotis, A.V.: Architecture independent parallel binomial tree option price valuations. Parallel Computing 30(2), 303-318 (2004)

8. Gerbessiotis, A.V.: Trinomial-tree based parallel option price valuations. Parallel Algorithms and Applications 18(3), 181-196 (2003)

9. Campolieti, G., Makarov, R.: Parallel lattice implementation for option pricing under mixed state-dependent volatility models. In: 19th International Symposium on High Performance Computing Systems and Applications (HPCS 2005), pp. 170176. IEEE Computer Society, Los Alamitos (2005)

10. Huang, K., Thulasiram, R.K.: Parallel algorithm for pricing american asian options with multi-dimensional assets. In: 19th International Symposium on High Performance Computing Systems and Applications (HPCS 2005), pp. 177-185. IEEE Computer Society Press, Los Alamitos (2005)

11. Moritsch, H., Benkner, S.: High-performance numerical pricing methods. Concurrency and Computation: Practice and Experience 14(8-9), 665-678 (2002)

12. Harris, D.: Will financial services drive Grid adoption? Grid Today 3(40) (2004)

13. Hull, J., White, A.: The general Hull-White model and super calibration. Financial Analysts Journal 57(6) (2001)

14. Dongarra, J., Sterling, T., Simon, H., Strohmaier, E.: High-performance computing: Clusters, constellations, MPPs, and future directions. Computing in Science and Engineering 7(2), 51-59 (2005) 